\title{
Formulation and evaluation of dexamethasone loaded stearic acid nanoparticles by hot homogenization method
}

\author{
*Shumaia Parvin'1, Md. Abu Shuaib Rafshanjani², Md. Abdul Kader ${ }^{1}$ \\ ${ }^{1}$ Department of Pharmacy, University of Rajshahi, Rajshahi 6205, Bangladesh \\ ${ }^{2}$ Department of Pharmacy, North South University, Plot 15, Block B, Bashundhara R/A, Dhaka-1229, Bangladesh
}

\begin{abstract}
Dexamethasone is a type of steroid medication having anti-inflammatory and immunosuppressant effects. One of the major problems with this drug is its low solubility in water which results into poor bioavailability after oral administration. So the objective of the present work is to improve the solubility and dissolution rate of dexamethasone using its solid lipid nano particles (SLNPs) with stearic acid as solid lipid, lutrol F-68 as surfactant and tween-80 as stabilizer. SLNPs are prepared by hot homogenization method at different ratio of drug, lipid, surfactant and stabilizer and designated as DNP1 to DNP6. In vitro dissolution study was performed using the USP type II apparatus (paddle method) at $50 \mathrm{rpm}$ to a temperature of $37^{\circ} \pm 0.5^{\circ} \mathrm{C}$ in distilled water containing $0.75 \% \mathrm{w} / \mathrm{v}$ SLS (sodium lauryl sulfate). The absorbance of sample was measured spectrophotometrically at $\lambda_{\max } 239 \mathrm{~nm}$ on a UV-Visible spectrophotometer. Release pattern of drug was found to follow zero order, first order and Korsmeyer-Peppas equations. Improvement of dissolution was observed in all the solid lipid nano particles as compared to pure drug. Pure drug showed only $27.25 \%$ release in $50 \mathrm{~min}$ whereas the dexamethasone SLNPs showed faster $(66.19 \%)$ in vitro drug release. Hence, this finding indicates that dexamethasone SLNPs prepared by hot homogenization method can be used to enhance the dissolution rate and to show novel application to this drug delivery system.
\end{abstract}

Key Words: Dexamethasone, poor water solubility, SLNPs, dissolution, release kinetics.

\section{INTRODUCTION}

In recent years, the number of active agents having low aqueous solubility increased significantly. Oral delivery of poorly water soluble drugs often results in low bioavailability since the rate-limiting step for absorption from the gastrointestinal tract is a significantly slower dissolution rate (Mitchell et al., 2003). Among the five key physicochemical properties in the early compound screening including pka, solubility, permeability, stability and lipophilicity, poor solubility tops the list of undesirable compound properties (Singh et al., 2010). Compounds with insufficient solubility carry a higher risk of failure during discovery and development, since it may compromise other properties of compound and add undesirable properties, can influence both pharmacokinetic and pharmacodynamic properties of the compound and finally may affect the bioavailability (Alsenz and Kansy, 2007; Sharma et al., 2013). Thus, many molecules that are biologically active in vitro are inactive in vivo as a result of their limited solubility and slow rate of dissolution (Ficarra et al., 2000; Zingone and Rubessa, 2005). So, formulation approaches are being improved to enhance bioavailability of poorly water-soluble drugs. One such approach to significantly enhance dissolution of such drugs is to prepare solid lipid nanoparticles. SLNs are sub-micron size colloidal carriers ranging from 50 to 1000 $\mathrm{nm}$, which are composed of physiological lipid, have been dispersed in water or in aqueous surfactant solution. SLNs possess solid lipid core matrix and solubilize lipophilic molecules (Waghmare et al., 2012). It has been reported that the surfactant molecules stabilize the lipid

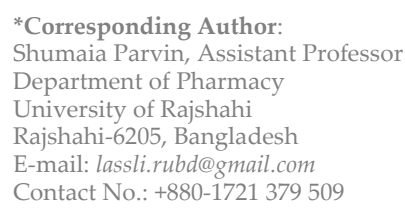

core and might prevent particle agglomeration more efficiently (Rawat et al., 2011). SLNs offer unique properties such as small size, large surface area, high drug loading and the interaction of phases at the interface and are attractive for their potential to improve performance of pharmaceuticals (Ekambaram et al., 2012; Mozafari, 2006). Due to biodegradable and bioacceptable nature of SLN these are less toxic than polymeric nanoparticles and it also overcomes some disadvantages of traditional colloidal drug carrier system (Nikam et al., 2014).

Dexamethasone (figure 1) is a synthetic adrenocortical steroid and is chemically designated as 9-fluoro- $11 \beta$, 17, 21-trihydroxy-16 $\alpha$-methylpregna-1, 4-diene, 3, 20dione. The drug is 25 times more potent than cortisol in its glucocorticoid effect, while having minimal mineralocorticoid effect. It has anti-inflammatory and immunosuppressant effects and is used for the treatment of many conditions including rheumatologic problems, a number of skin diseases such as erythema multiforme, severe allergies, asthma, chronic obstructive lung disease, croup, cerebral edema, in addition to other medications in tuberculosis and a number of other infectious diseases. However, it is characterized by a low water solubility $\left(0.08 \mathrm{mg} / \mathrm{ml}\right.$ at $\left.25^{\circ} \mathrm{C}\right)$ and consequently low and irregular bioavailability (Swartz and Dluhy, 1978). Also the therapeutic efficacy has been questioned with regard to its short half-life (O'Byrne and Pederson, 1998), potential toxicity at high doses (Foster et al., 2006). Therefore, some advanced delivery systems of drug need to be developed. Hence, in present experiment we, herein report about preparation of dexamethasone-loaded solid lipid nano particles with stearic as solid lipid and evaluation of enhanced dissolution rate as well as improved bioavailability. 


\section{MATERIALS AND METHODS}

\section{Materials}

Dexamethasone was a gift sample from Square Pharmaceuticals Ltd, Dhaka, Bangladesh. Stearic acid was procured from Balami Fine Chemicals, India. Tween- 80 and lutrol F-68 (Poloxamer) were received from BASF, Germany. All other reagents and solvent used were of analytical grade.

\section{Methods}

Preparation of dexamethasone SLNPs by hot homogenization method

The solid lipid nanoparticles of dexamethasone were prepared using the lipid (stearic acid); surfactant (lutrol F68) and stabilizer (tween-80) at different proportion by hot homogenization technique (Mehnert and Mader, 2001; Kumar et al., 2012; Jahnke, 1998; Ahlim et al., 1998; Vyas and Khar, 2006). At first dexamethasone was dissolved in acetone and the lipid was melted through hot plate by maintaining the temperature above the melting point $69.6^{\circ} \mathrm{C}$ of the lipid. The dissolved drug was mixed with melted lipid and heated until the evaporation of acetone occurs. Now surfactant and stabilizer were heated with distilled water on hot plate at the same temperature of lipid mixture. Hot surfactant solution was then added to the melted lipid-drug mixture and emulsified by a homogenizer (M-110P lab homogenizer, Trident Equipment's Pvt. Ltd., Mumbai, India) at $12000 \mathrm{rpm}$ for 2 hours. The formulation was then removed from water bath and finally the dispersion of SLNPs was mixed gently at room temperature (table 1).

\section{In vitro dissolution study of dexamethasone solid lipid nanoparticles}

In vitro dissolution studies of dexamethasone SLNPs were carried out to estimate the cumulative percentage of drug release with respect to time. $900 \mathrm{ml}$ distilled water containing $0.75 \% \mathrm{w} / \mathrm{v}$ SLS was placed in each vessel of the USP type II rotating paddle dissolution apparatus (Veego Vda 6DR, Germany). SLNPs were placed in each vessel and the medium was allowed to maintain at $50 \mathrm{rpm}$ to a temperature of $37^{\circ} \pm 0.5^{\circ} \mathrm{C}$ for 50 minutes. $5 \mathrm{ml}$ of the sample was withdrawn at definite time interval $(10,20,30$, 40 and $50 \mathrm{~min}$ ) consecutively and replaced immediately by $5 \mathrm{ml}$ of fresh media. The absorbance of sample was measured spectrophotometrically at $\lambda_{\max } 239 \mathrm{~nm}$ on a UVVisible spectrophotometer (UVmini-1240, Shimadzu Corp., Kyoto, Japan) and the values of dissolution efficiency were calculated (Khan and Rhodes, 1975).

Table 1: Formulation of dexamethasone SLNPs through hot homogenization method using various ratios of lipid, surfactant and stabilizer.

\begin{tabular}{|c|c|c|c|c|c|c|}
\hline 总全 & 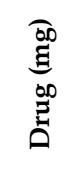 & 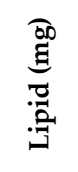 & 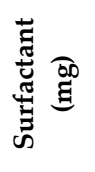 & 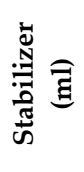 & 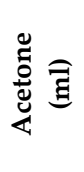 & 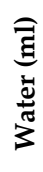 \\
\hline DNP1 (1:2:1:1) & 1000 & 2000 & 1000 & 1 & 20 & 20 \\
\hline DNP2 (1:2:1:1.5) & 1000 & 2000 & 1000 & 1.5 & 20 & 20 \\
\hline DNP3 (1:1:1:2) & 1000 & 1000 & 1000 & 2 & 20 & 20 \\
\hline DNP4 (1:2:2:1) & 1000 & 2000 & 2000 & 1 & 20 & 20 \\
\hline DNP5 (1:2:2:1.5) & 1000 & 2000 & 2000 & 1.5 & 20 & 20 \\
\hline DNP6 (1:2:2:2) & 1000 & 2000 & 2000 & 2 & 20 & 20 \\
\hline
\end{tabular}

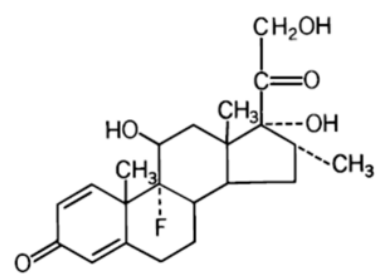

Figure 1: Structural formula of dexamethasone.

\section{Release kinetics of dexamethasone from solid lipid nano particles}

Data obtained from drug release studies were plotted in various kinetics; zero order (equation 1) as cumulative amount of drug released vs. time, first order (equation 2) as log cumulative percentage of drug remaining vs. time and Korsmeyer-Peppas model as log cumulative percentage of drug released vs. log time (equation 3 ).

$\mathrm{Ct}=\mathrm{C}_{0}+\mathrm{K}_{0} \mathrm{t} \quad \ldots .(\mathbf{1})$

Where, $\mathrm{K}_{0}$ is the zero order rate constant expressed in units of concentration/time and $t$ is the release time. A graph of concentration vs time would yield a straight line with a slope equal to $K_{0}$ and intercept the origin of the axes (Hadjiioannou et al., 1993)

$$
\log C=\log C_{0}-\frac{k t}{2.303} \quad \ldots . \text { (2) }
$$

Where, $\mathrm{C}_{0}$ is the initial concentration of drug, $\mathrm{k}$ is the first order rate constant and $t$ is the time (Bourne, 2002)

$$
\log \left(\frac{M_{t}}{M_{f}}\right)=\log k+n \log t \quad \ldots . \text { (3) }
$$

Where, $M_{t}$ is the amount of drug release at time $t, M_{f}$ is the amount of drug release after infinite time, $t$ is the release time, $\mathrm{K}$ is the kinetic constant characteristic of the drug polymer/system and $\mathrm{n}$ is an exponent that characterizes the mechanism of release of drug (Korsmeyer et al., 1983). A value of $n=0.45$ indicates Fickian diffusion (case-I) release; $0.45<\mathrm{n}<0.89$ for non-Fickian diffusion (anomalous) release; $n=0.89$ case-II transport and $n>0.89$ indicates super case II release.

\section{RESULTS AND DISCUSSION}

The results of the dissolution studies are summarized in terms of percentage release of dexamethasone in $50 \mathrm{~min}$ period of time and also release kinetic modeling of drug from SLNPs. The drug release data were extrapolated by zero order, first order and Korsmeyer-Peppas equations as depicted by figure 2, 3 and 4 . Regression coefficient $\left(\mathrm{r}^{2}\right)$ calculated from the slope of the plot and diffusion exponent ' $n$ ' are enlisted in table 2. For this system first order kinetic model yielded remarkably better linearity

Table 2: Regression coefficient $\left(\mathrm{r}^{2}\right)$ of different kinetic model and diffusion exponent (n) of Korsmeyer-Peppas model of dexamethasone release from different SLNP formulations.

\begin{tabular}{ccccc}
\hline $\begin{array}{c}\text { Formula- } \\
\text { tion code }\end{array}$ & $\begin{array}{c}\text { Zero order } \\
\text { model }\left(\mathbf{R}^{2}\right)\end{array}$ & $\begin{array}{c}\text { First order } \\
\text { model (R }\end{array}$ & \multicolumn{2}{c}{ Korsmeyer-Peppas model } \\
\cline { 4 - 5 } DNP1 & 0.994 & 0.978 & $\mathbf{R}^{\mathbf{2}}$ & $\mathbf{N}$ \\
\hline DNP2 & 0.883 & 0.981 & 0.978 & 0.861 \\
DNP3 & 0.998 & 0.931 & 0.993 & 0.455 \\
DNP4 & 0.988 & 0.979 & 0.996 & 0.455 \\
DNP5 & 0.955 & 0.969 & 0.965 & 0.842 \\
DNP6 & 0.981 & 0.987 & 0.982 & 0.999 \\
\hline
\end{tabular}




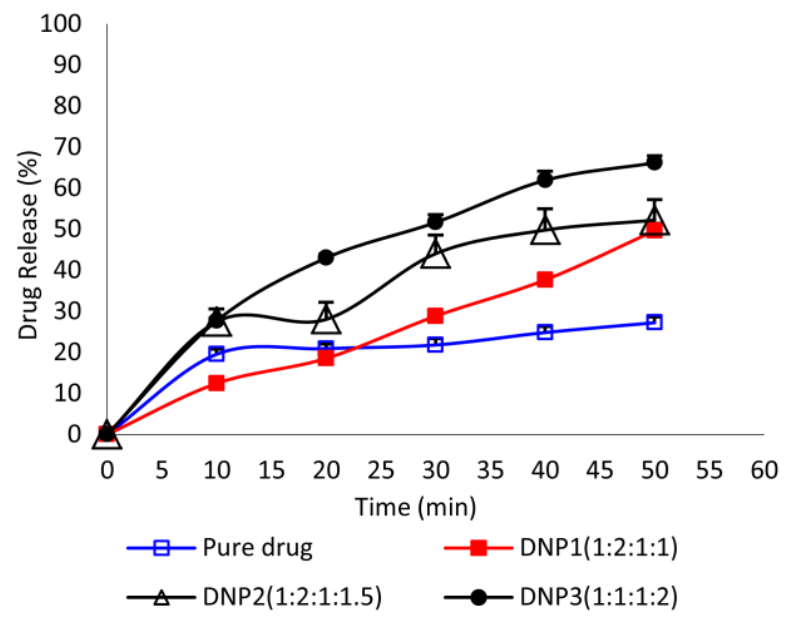

Figure 2(a): Zero order plot for release kinetics of dexamethasone from different SLNPs (DNP1, DNP2 and DNP3).

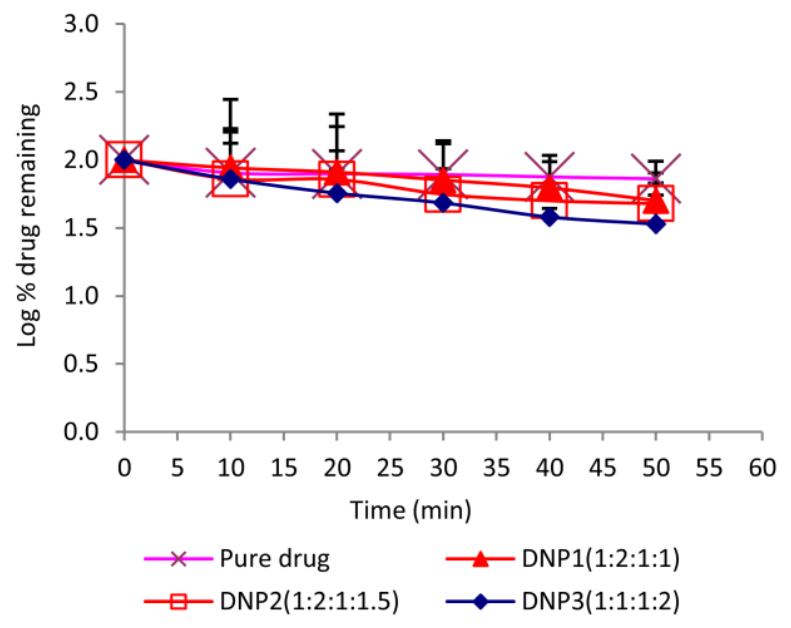

Figure 3(a): First order plot for release kinetics of dexamethasone from different SLNPs (DNP1, DNP2 and DNP3).

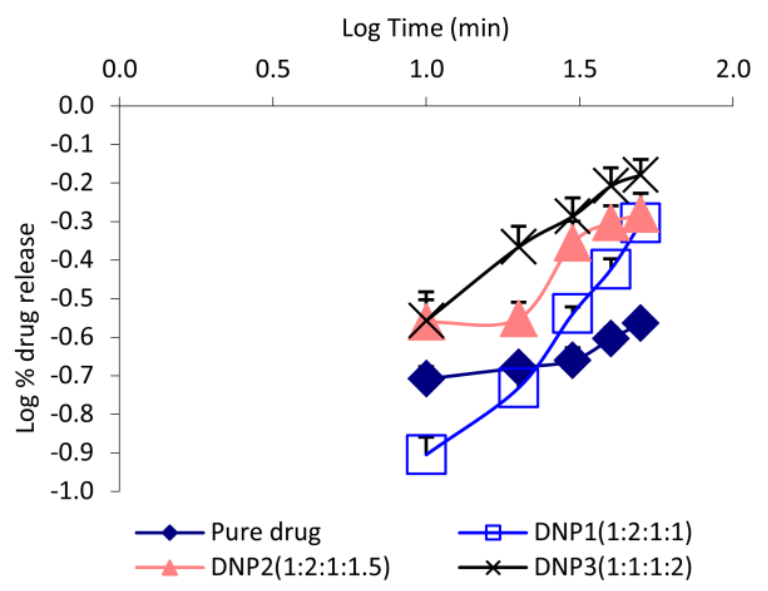

Figure 4(a): Korsmeyer Peppas plot for release kinetics of dexamethasone from different SLNPs (DNP1, DNP2 and DNP3).

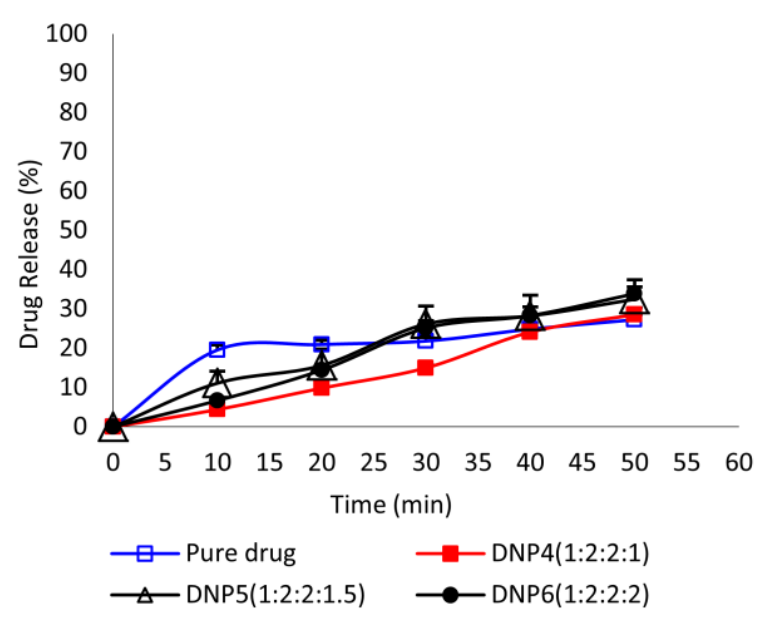

Figure 2(b): Zero order plot for release kinetics of dexamethasone from different SLNPs (DNP4, DNP5 and DNP6).

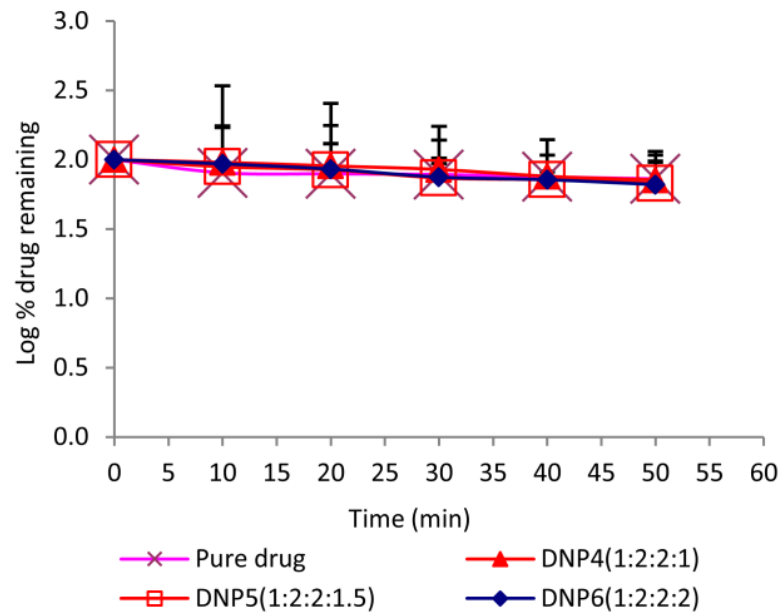

Figure 3(b): First order plot for release kinetics of dexamethasone from different SLNPs (DNP4, DNP5 and DNP6).

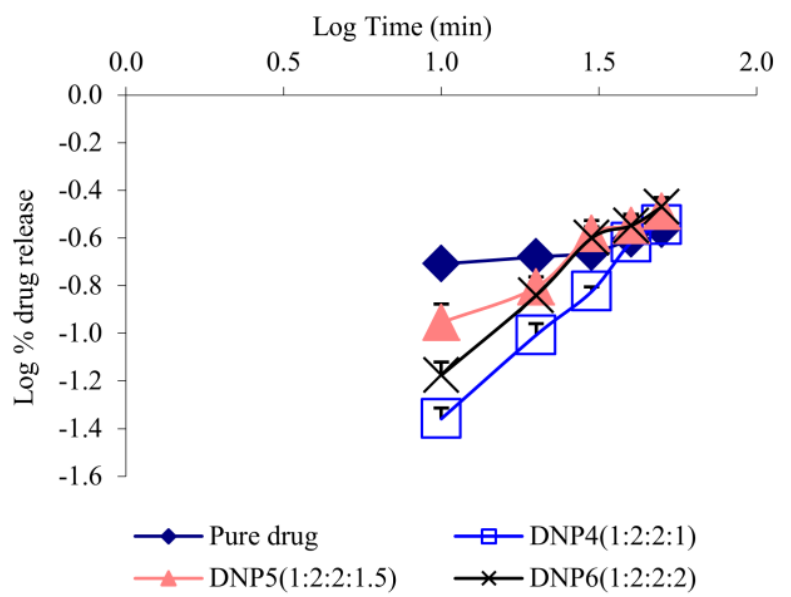

Figure 4(b): Korsmeyer Peppas plot for release kinetics of dexamethasone from different SLNPs (DNP4, DNP5 and DNP6). 


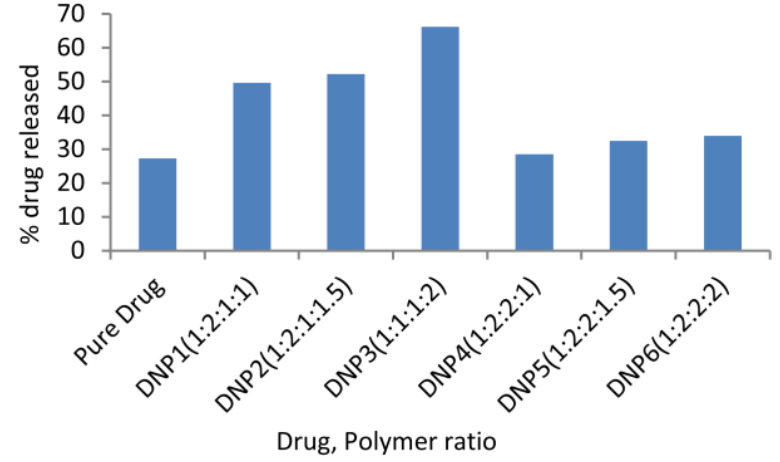

Figure 5: Graphical representations for drug release efficiency of dexamethasone from different SLNPs as compared to pure drug (DNP1, DNP2, DNP3, DNP4, DNP5 and DNP6).

with $\mathrm{r}^{2} 0.931$ to 0.987 . Moreover, zero order and Korsmeyer-Peppas model yielded similarly good quality adjustments with $r^{2} 0.883$ to 0.998 and $r^{2} 0.855$ to 0.996 respectively. The analysis of experimental data in the light of the Korsmeyer-Peppas equation as well as interpretation of the corresponding release exponent ' $n$ ' leads to a better understanding of the release mechanism of drug. It is evident from the table 2 that ' $n$ ' values vary from 0.455 to 0.928 which is pointing to an anomalous (non-Fickian) diffusion mechanism. It may indicate that the drug release is controlled by more than one process that is a coupling of the diffusion and erosion mechanism. Researchers Fassihi and Ritschel also observed the similar results with a matrix tablet of theophylline with an ' $\mathrm{n}$ ' value of 0.7 (Fassihi and Ritchel, 1993).

Formulated SLNPs exhibited significant improvement in the dissolution parameter of dexamethasone illustrated by figure 5 . The order of dissolution enhancement with various SLNP systems was found to be DNP3 $>$ DNP2 $>$ DNP1 $>$ DNP6 $>$ DNP5 $>$ DNP4. As is evident from the Figure 5 the dissolution rate of DNP3 is higher $(66.19 \%)$ and that of pure drug is lower $(27.25 \%)$ even in $0.75 \% \mathrm{w} / \mathrm{v}$ SLS containing medium. Even when the percentage of drug release of this optimized formulation, DNP3 was compared with market preparation and pure drug (figure 6), optimized formulation showed highest release $(66.19 \%)$ than market preparation $(41.82 \%)$.

The increase in dissolution rate is probably due to some factors, when lipid stearic acid is used to prepare SLNPs by homogenization method, this process converts the solid state of the drug from a crystalline material to an amorphous high energy state and dexamethasone is wetted more effectively by dissolution media with a greater dissolution rate. This lipid also increases the entrapment efficiency of dexamethasone by increasing the viscosity of medium which prevents rapid diffusion of drug into bulk of medium (Yang et al., 2000). The high pressure homogenizer also helps to achieve a further reduction in particle size which is also credited to higher dissolution rate. During the in vitro dissolution assay, surfactants act in an important role to maintain the stability of SLNPs (Dong-Hwan and Martin, 2006). When dexamethasone is released from SLNPs, the surfactant attaches to the hydrophobic nano particles surface and prevents the drug from diffusing back into the solid core of nano particles. Probably, all these factors could contribute together resulting in increased solubility and

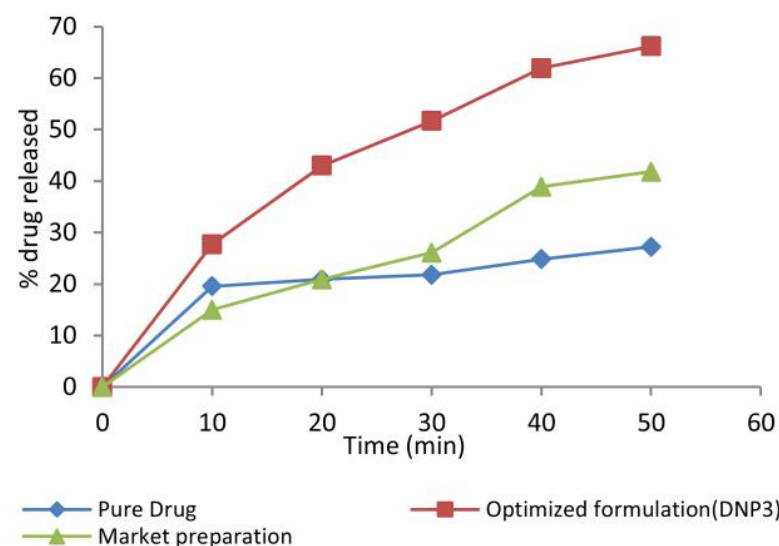

Figure 6: Optimization of release profile of dexamethasone SLNPs (pure drug, optimized formulation; DNP3 and market preparation).

dissolution properties of dexamethasone solid lipid nano particles.

\section{CONCLUSION}

The therapeutic window of dexamethasone is narrow, so it is necessary to develop a SLNP system that releases the drug within this window. Thus, the formulation DNP3 with 1:1:1:2 ratio of drug, lipid, surfactant and stabilizer was considered better for this system, can be designed to improve dissolution rate and bioavailability of dexamethasone.

\section{ACKNOWLEDGEMENT}

The authors are grateful to Square Pharmaceuticals Ltd, Dhaka, Bangladesh for supplying the gift sample of dexamethasone and to the Department of Pharmacy, University of Rajshahi, Rajshahi 6205, Bangladesh for providing laboratory facilities. This work was also supported by the Department of Pharmacy, North South University, Plot 15, Block B, Bashundhara R/A, Dhaka1229, Bangladesh.

\section{REFERENCES}

Ahlim, P. G., Kristl, J. and Korbar, J. S. (1998). Optimization of procedure parameters and physical stability of solid lipid nano particles in dispersion. Acta Pharmaceutica, Volume 48, Pages 259-267.

Alsenz, J. and Kansy, M. (2007). Thermodynamic solubility assay. Advanced Drug Delivery Reviews, Volume 59, Pages 546-556.

Bourne, D.W. (2002). Pharmacokinetics. Banker, G.S. and Rhodes, C.T. editors. Modern Pharmaceutics (4th ed., pp. 67-92). New York, Marcel Dekker Inc.

Dong-Hwan, K. and Martin D.C. (2006). Sustained release of dexamethasone from hydrophilic matrices using PLGA nano particles for neural drug delivery. Biomaterial, Volume 27, Issue 15, Pages 3031 3037. [DOI]

Ekambaram, P., Sathali, A.A.H. and Priyanka, K. (2012). Solid lipid nanoparticles: A review. Scientific Reviews and Chemical Communications, Volume 2, Issue 1, Pages 80-102.

Fassihi, R.A. and Ritschel, W.A. (1993). Multiple layer, direct compression controlled release system: in vitro and in vivo evaluation. Journal of Pharmaceutical Science, Volume 82, Pages 750-754. [DOI]

Ficarra, R., Ficarra, P. and Bella, M.R.D., Raneri, D., Tommasini, S., Calabro, M.L., Villari, A. and Coppolino S (2000). Study of the inclusion complex of atenolol with $\beta$-cyclodextrins. Journal of Pharmaceutical and Biomedical Analysis, Volume 23, Issue I, Pages 231-236. [DOI]

Foster, J.M., Aucott, L., van der Werf, R.H., van der Meijden, M.J., Schraa, G., Postma, D.S. and van der Molten, T. (2006). Higher patient perceived side effects related to higher daily doses of inhaled 
corticosteroids in the community: a cross-sectional analysis. Respiratory Medicine, Volume 100, Issue 8, Pages 1318-1336. [DOI]

Hadjiioannou, T.P., Christian, G.D. and Koupparis, M.A. (1993) Quantitative Calculations in Pharmaceutical Practice and Research (pp. 345-348). New York, VCH Publishers Inc.

Jahnke, S. (1998). The theory of high pressure homogenization. Muller R.H., Benita, S. and Bohm, B. editors. Emulsions and nano suspensions for the formulation of poorly soluble drugs (pp. 77). Stuttgart, Medpharm Scientific publishers.

Khan, C.A. and Rhodes, C.T. (1975). The concept of dissolution efficiency. Journal of Pharmacy and Pharmacology, Volume 27, Issue 1, Pages 48 49. [DOI]

Korsmeyer, R.W., Gurry, R., Doelker, E., Buri, P. and Peppas, N.A. (1983). Mechanisms of solute release from porous hydrophilic polymers. International Journal of Pharmacy, Volume 15, Issue 1, Pages 25-35. [DOI]

Kumar, P.D., Subas, D.C., Subrata, C. and Soumen, R. (2012). Formulation and evaluation of solid lipid nano particles of a poorly water soluble model drug ibuprofen. International Research Journal of Pharmacy, Volume 3, Issue 12, Pages 132-137.

Mehnert, W. and Mader, K. (2001). Solid lipid nanoparticles production, characterization, and applications. Advance Drug Delivery and Review, Volume 47, Issues 2-3, Pages 165-196. [DOI]

Mitchell, S.A., Reynolds, T.D. and Dasbach, T.P. (2003). A compaction process to enhance dissolution of poorly water soluble-drugs using hydroxypropyl methylcellulose. International Journal of Pharmacy, Volume 250, Issue 1, Pages 3-11. [DOI]

Mozafari, M.R. (2006). Nano carrier technologies. Frontiers of Nano Therapy, Pages 41-50.

Nikam, S., Chaven, M. and Sharma, P.H. (2014). Solid lipid nanoparticles: A lipid based drug delivery. Innovations in Pharmaceuticals and Pharmacotherapy, Volume 2, Issue 3, Pages 365-376.
O'Byrne, P.M. and Pederson S. (1998). Measuring efficacy and safety of different inhaled corticosteroid preparations. Journal of Allergy and Clinical Immunology, Volume 102, Issue 6, Pages 879-886. [DOI]

Rawat, M.K., Jain, A. and Singh S. (2011). Studies on binary lipid matrix based solid lipid nano particles of repaglinide: in-vitro and in-vivo evaluation. Journal of Pharmaceutical Sciences, Volume 100, Issue 6, Pages 2366-2378. [DOI]

Sharma, M., Rajeev, G. and Gupta, G.D. (2013). Formulation and evaluation of solid dispersion of atorvastatin calcium. Journal of Pharmaceutical and Scientific Innovation, Volume 2, Issue 4, Pages 7381. [DOI]

Singh, M., Sayyad, A.B. and Sawant, S.D. (2010). Review on various techniques of solubility enhancement of poorly soluble drugs with special emphasis on solid dispersion. Journal of Pharmacy Research, Volume 3, Issue 10, Pages 2494-2501.

Swartz, S.L. and Dluhy, R.G. (1978). Corticosteroids: clinical pharmacology and therapeutic use. Drugs, Volume 16, Pages 238-255.

Vyas, S.P. and Khar, R.K. (2006). Targeted and Controlled Drug Delivery Novel Carrier System (1st ed., pp. 15-16). CBS Publication.

Waghmare, A.S., Grampurohit, N.D., Gadhave M.B., Gaikwad, D.D. and Jadhav, S.L. (2012). Solid Lipid Nanoparticles: A promising drug delivery system. International Research Journal of Pharmacy, Volume 3, Issue 4, Pages 100-107.

Yang, Y., Chung, T., Bai, X. and Chan, W. (2000). Effect of preparation conditions on morphology and release profiles of biodegradable polymeric microspheres containing protein fabricated by doubleemulsion method. Chemical Engineering and Science, Volume 55, Issue 12, Pages 2223-2236. [DOI]

Zingone, G. and Rubessa, F. (2005). Preformulation study of the inclusion complex warfarin-B-cyclodextrin. International Journal of Pharmacy, Volume 291, Issues 1-2, Pages 3-10. [DOI] 